Egyptian

Orthodontic Journal

\title{
DENTOFACIAL CHANGES ASSOCIATED WITH UNIARCH EXTRACTION TREATMENT OF CLASS II MALOCCLUSION
}

\author{
Maher A Fouda*, Ahmed A El-bialy", \\ Yasser L A Mahmoud ${ }^{* * *}$ and Ghada. A. El.Mehy ${ }^{* * *}$
}

ABSTRACT

This study was carried-out to evaluate the changes of the dental and skeletal variables accompanied with the uni-arch extraction treatment of Class II div 1 malocclusions.

The sample of this study was consisted of twelve female patients at the beginning of treatment and ended by ten patients due to sample decay. Full fixed edgewise appliances were fitted, and then the upper canines were retracted after two weeks from upper first premolars extraction. The upper incisors were retracted using closing arches with high pull headgears. The lower arches were treated without extraction, just aligned and finished. For each patient pre and post orthodontic casts and lateral cephalometric roentgenograms were taken. The collected data were managed using SPSS statistical program to get Paired test.

The outcome revealed decrease in the maxillary arch perimeters (t-value $=17.08)$, increase the mean changes of upper inter-canine widths( $t$-value $=4,4$ ) and significant reduction in 6oth the overjet as well as the overbite as measured on the casts. The maxillary cephalometric changes were significantly decreased, SNA Angle, effective maxillary length and inclination of upper incisors relative to

\footnotetext{
* Professor of Orthodontics, Faculty of Dentistry, Mansoura University, Egypt

${ }^{* *}$ Associate Professor Orthodontics, Faculty of Dentistry, Mansoura University, Egypt

${ }^{* * *}$ Lecturer of Orthodontics, Faculty of Dentistry, Mansoura University, Egypt.

${ }^{* * * *}$ Lecturer of Orthodontics, Faculty of Dentistry, Tanta University, Egypt.
} 
Egyptian

Orthodontic Journal

FH plane. These changes were not significant for the lower arch except the IMMPA angle. The maxillary/mandibular relationships were also affected as there were significant reduction in both $\mathcal{A N} B$ angle and overjet.

\section{INTRODUCTION}

Class II malocclusion is one of the most common malocclusions. It represents about $21 \%$ of Egyptian adult malocclusions ${ }^{(1)}$. The major treatment objective is to establish a stable occlusion. Treatment of Class II malocclusion may be performed by either extraction or non extraction methods ${ }^{(2-5)}$. Some compliance treatment modalities has been hampered by the use of appliances which require patient cooperation with headgear and Class II elastics. Others used the non-compliance therapy through the use of appliances which minimize the need for such cooperation and attempt to maximize the predictability of results ${ }^{(6)}$.

In forty-years review of extraction frequencies to camouflage of Class II malocclusions, Proffit ${ }^{(7)}$ found that extraction of maxillary first premolars alone reached $16 \%$ in 1968, then declined. However, presently it is increased but not as frequent as the extraction of four first premolars. According to Conley and Jernigan(8) the maxillary first premolar extraction for orthodontic camouflage of Class II is now available as a treatment option. So, the goal of finishing with Class II molars and Class I canines is a variable functional compromise ${ }^{(9)}$.

According to D. $\operatorname{Root}^{(10)}$ on a roundtable, extractions depend on a certain extent to the type of case, high or low mandibular angle and whether the patient is growing or adult. Generally, with medium crowding, he extracted upper first bicuspids only, but in the cases with minimum crowding, he extracted all four second bicuspids or upper first and lower second bicuspids. However, significant changes were found in arch parameters such as intermolar width in the extraction compared to nonextraction groups of Class II division1 malocclusions ${ }^{(11-13)}$. In general, the trends in the post-treatment changes were similar in males and females as well as in the maxillary and mandibular arches. The arch perimeters were also, decreased while the intercanine widths were significantly increased after canine retractions ${ }^{(14)}$.

The maxillary canines occupy a unique position in the dental arch and thus considered of prime importance ${ }^{(15)}$. The correct position of the canine eminences at the corners between the anterior and posterior segments seems to have a very important role in maintaining facial expression and skeletal balance. Despite of 
seriousness of uniarch extraction and canine retraction in one arch in many patients there is no available data on the effect of uniarch extraction modalities; uniarch canine retraction and alteration of the canine eminences position during their retractions on the dental parameters e.g., intercanine width and arch perimeter, overjet, overbite and also; the skeletal framework.

The purpose of this study was to evaluate the changes of the dental and skeletal variables accompanied with the uni-arch extraction treatment of Class II division 1 malocclusions.

\section{Materials and Methods}

\section{Sample:}

The sample of this study was consisted of twelve female patients at the beginning of treatment and ended by ten patients due to sample decay. They were selected from the outpatient clinic of Orthodontic Department, Mansoura Faculty of Dentistry. Their age ranged from 11.4 to 13.8 years. The patients were selected according to the following criteria:-

Class II division 1 or a subdivision with increased overjet(4- $7 \mathrm{~mm})$ and Class II canines relationships.

The lower arch was well aligned or with minimal crowding that would allow non-extraction treatment.

- All patients were in early permanent dentition stage.

None of the patient suffered from undesirable oral habits and had no history of previous orthodontic treatment.

\section{Method:}

For each patient the following records were taken ; orthodontic casts and lateral cephalometric roentgenograms. Full fixed edgewise appliances were fitted, then the upper canines were retracted after alignments along an .018" stainless steel round wire to avoid deformation and with enough clearance in an .022 " standard edgewise brackets to avoid friction (16). The upper incisors retracted using closing arches and high pull headgear. The lower arches were treated without extraction, just aligned and finished.

\section{1-Dental cast measurements:}

Arch perimeter; the distance from the mesial contact point of the first molar to the mesial contact point of the other first molar passing over the buccal cusps of premolars, and the incisal edge of incisors . 
Inter-canine width; is the distance between the cusp tips of the canines or estimated cusp tips in cases of wear facets

Overjet; the horizontal distance from the labial incisal edge of the most protruded upper incisor to the labial surface of the opposing mandibular central incisor tooth.

Overbite; the vertical overlap of the lower incisors by the upper incisors.

\section{Cephalometric analysis:}

Cephalometric films were traced and the following landmarks were identified (17): Sella Turcica(S), Nasion(N), Porion(P), Orbitale (Or), point A (A), point B (B), Menton (Me), anterior nal spine (ANS), Posterior nasal spine (PNS), Pogonion (PG), gnathion (Gn), Articulare (Ar) and center of the condyle (Condylion). Then the lines and planes were drawn on the tracing and linear and angular measurements were calculated.

\section{Cephalometric Measurements:}

SNA; Describes the sagittal position of maxillary apical base relative to the anterior cranial base and indicates horizontal location of the maxilla

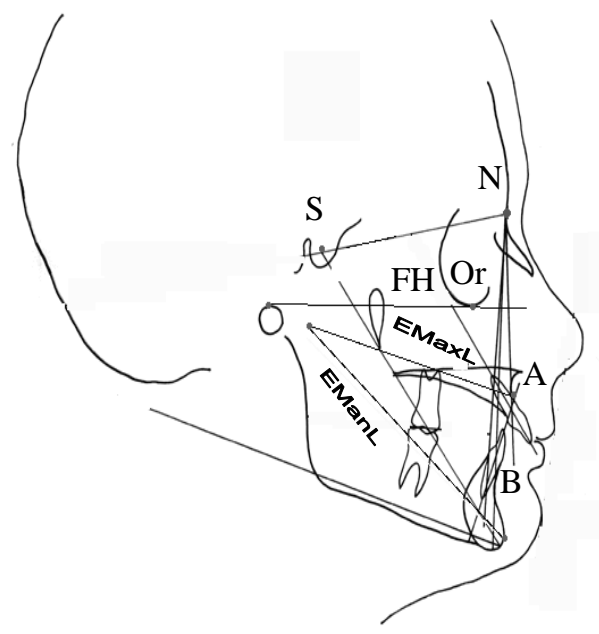

Fig. (1): Cephalometric points and planes 
SNB; Describes the sagittal position of the mandibular apical base relative to the anterior cranial base.

ANB; It is a three points angular measurements that describes the sagittal difference between the relative positions of the maxillary and mandibular apical bases. It is also used to describe the horizontal skeletal imbalance of the maxilla and mandible.

Upper incisor/FH (upp.inc./FH): It is a four point angular measurement which relates the upper incisor to the F-H plane .

IMPA; It is four- point angular measurement and defined as the angle of the long axis of the most protruded lower central incisor to mandibular plane .

Incisor Overjet (O.J); extent of the horizontal malocclusion measured at the incisors (18).

Effective mandibular length (eff. max. L); two point linear measurement, from condylion and Gnathion.

Effective maxillary length; two point linear measurement from condylion to point $\mathrm{A}$ and determines the effective midfacial length.

\section{Error of Method}

The tracing of the lateral head cephalograms were randomly selected and the linear and angular variables were measured then, they remeasured one month later and the mean of obsevations were recorded.

Statistical analysis of the collected data was done using SPSS program with a personal computer. The means and standard deviations for the pre and post-treatment measurements with the changes were calculated. Paired t-test at 0.05 level of significance was used to evaluate the significance of differences between pre and post-measurements.

\section{RESULTS}

Table 1 depicts the measurements to evaluate the dental treatment changes . Mean and standard deviation were calculated as well as paired t-test to display the treatment changes in the sample. In addition, the results were displayed graphically in figure 2 . 
Table 1: The means and standard deviations as well as the mean treatment changes of the cast measurements..

\begin{tabular}{|c|c|c|c|c|c|c|c|}
\hline \multirow{2}{*}{ Variables } & \multicolumn{2}{|c|}{ Pre-treatment } & \multicolumn{2}{c|}{ Post-treatment } & \multirow{2}{*}{ Mean } & \multicolumn{2}{c|}{ Significance } \\
\cline { 2 - 4 } & Mean & S.D. & Mean & S.D. & change & t. value & P $\leq$ \\
\hline UAP & 35.73 & S.D. & 29 & \pm 0.86 & 6.73 & 17.08 & $0.001^{* * *}$ \\
\hline LAP & 23.3 & \pm 1.85 & 23.33 & \pm 1.32 & 0.33 & 0.75 & 0.471 \\
\hline UICW & 22.5 & \pm 1.19 & 23.83 & \pm 1.63 & 1.33 & 4.43 & $0.002^{* * *}$ \\
\hline LICW & 21.8 & \pm 0.75 & 21.83 & \pm 1.63 & 0.33 & 2 & 0.81 \\
\hline Overjet & 6.5 & \pm 1.58 & 2.66 & \pm 0.25 & 3.83 & 12.75 & $0.001^{* * *}$ \\
\hline Overbite & 0.33 & \pm 0.75 & 0.27 & \pm 0.4 & 0.04 & 4 & $0.04^{*}$ \\
\hline
\end{tabular}

- UAP=upper arch perimeter.

- LAP=lower arch perimeter.

- UICW=upper inter canine width.

- LICW=lower inter canine width.

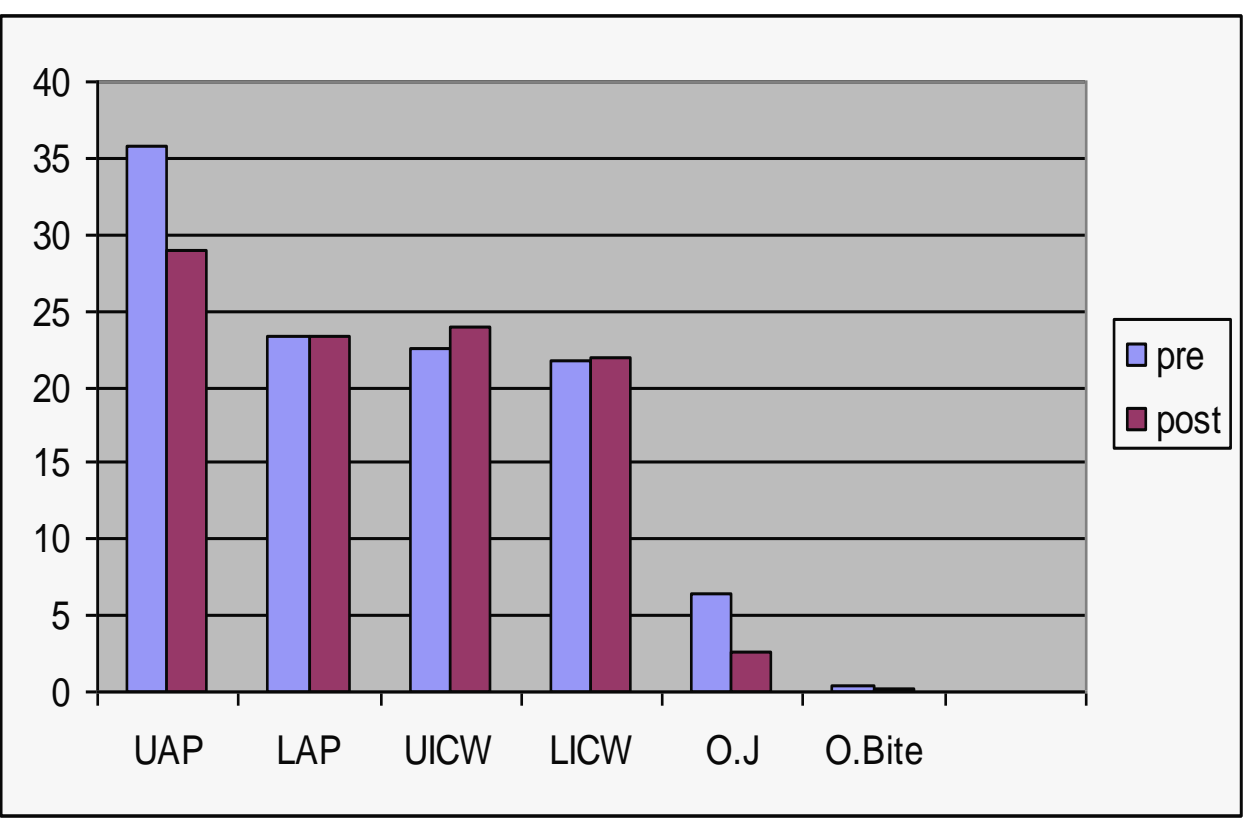

Figure 2: comparison between the means values of the dental variables measured on casts. 
The treatment changes were significant as there was decrease in upper arch perimeter and significant increase in the upper inter-canine width but there were no significant treatment changes of those parameters in the lower ones. There was also significant decrease in the overjet and overbite as measured on the casts.

Table 2 displays the treatment changes and their significance of the maxillary variables as measured on cephalograms. The mean and standard deviation were calculated as well as paired t-test to display the treatment changes in the sample. In addition, the results were displayed graphically in figure 3 .

Table 2: The means and standard deviations as well as well as the paired $t$ value of the maxillary treatment changes measured on cephalograms.

\begin{tabular}{|c|c|c|c|c|c|c|}
\hline \multirow{2}{*}{ Variables } & \multicolumn{2}{|c|}{ Pre-treatment } & Post-treatment & \multicolumn{2}{c|}{ Significance } \\
\cline { 2 - 7 } & Mean & S.D. & Mean & S.D. & P-t Value & P $\leq$ \\
\hline SNA & 83.92 & \pm 0.67 & 82.42 & \pm 0.6 & 13.74 & $0.001^{* * *}$ \\
\hline Eff. Max. L (In mm) & 88.14 & \pm 1.06 & 86.57 & \pm 1.09 & 5.28 & $0.002^{* * *}$ \\
\hline upp. inc. /FH & 26.78 & \pm 0.75 & 25.14 & \pm 1.02 & 7.81 & $0.001^{* * *}$ \\
\hline
\end{tabular}

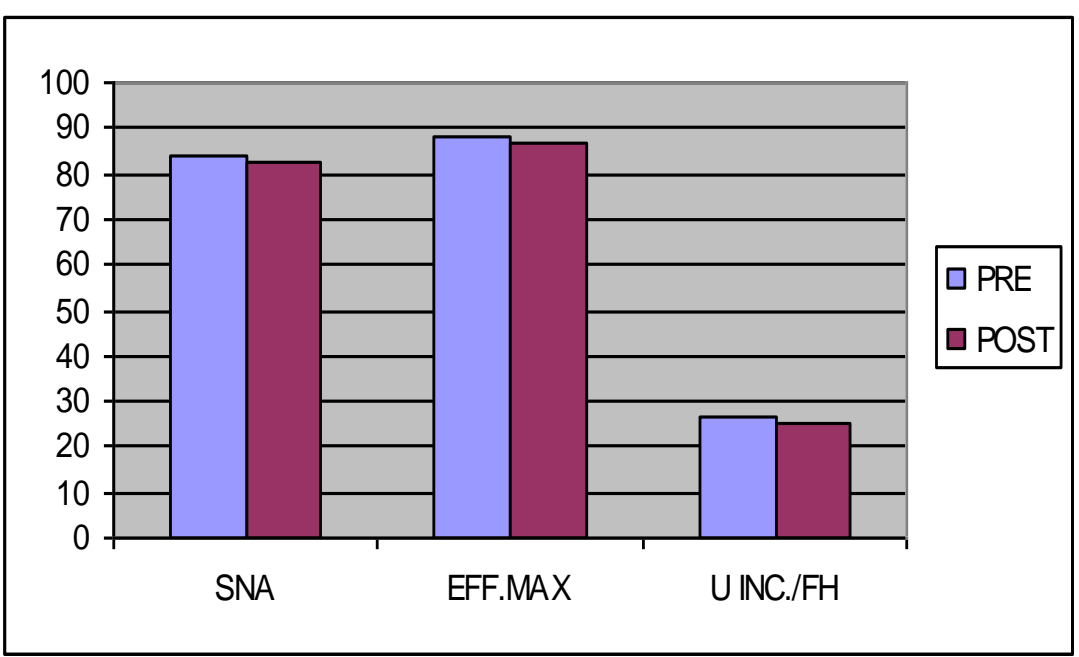

Figure 3: The mean changes were significant for all variables the S-N-A, Eff. Max. L and Upper incisor to FH. 
Table 3 and figure 4: illustrate the changes in the mandibular measurements and their significance as depicted from cephalograms. The mean and standard deviation were calculated as well as paired t-test to display the treatment changes in the sample. In addition, these results were showed graphically in figure 4. The S-N-B angle showed no significant changes as well as the effective mandibular length but there was significant increase in the relation of lower incisor to mandibular plane $(\mathrm{p}-\mathrm{t}=4)$.

Table 3: Means and standard deviations as well as the paired $t$ value of the mandibular treatment changes measured on cephalograms

\begin{tabular}{|c|c|c|c|c|c|c|}
\hline \multirow{2}{*}{ Variables } & \multicolumn{2}{|c|}{ Pre-treatment } & \multicolumn{2}{c|}{ Post-treatment } & \multicolumn{2}{c|}{ Significance } \\
\cline { 2 - 7 } & Mean & S.D. & Mean & S.D. & P-t Value & P $\leq$ \\
\hline SNB & 79.16 & \pm 0.76 & 79.5 & \pm 0.5 & 2 & 0.18 \\
\hline Eff. Max. L (In mm) & 108.66 & \pm 1.52 & 108.83 & \pm 1.75 & 1.02 & 0.42 \\
\hline IMPA & 97.33 & \pm 0.76 & 98 & \pm 0.5 & 4 & 0.057 \\
\hline
\end{tabular}

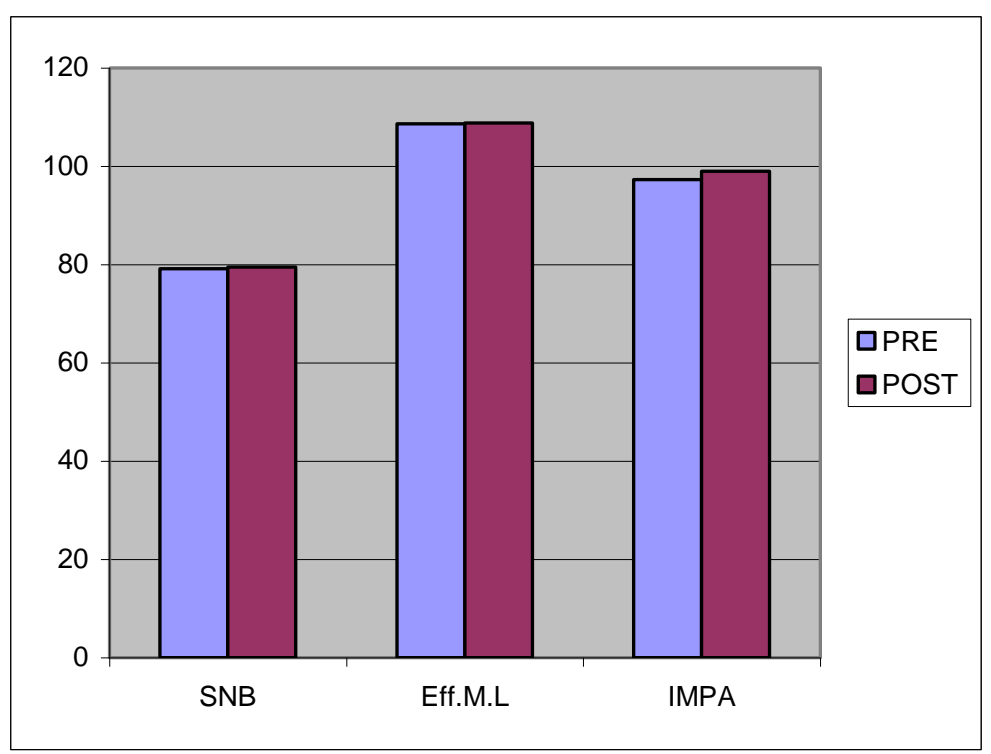

Figure 4: comparison between the means values of the mandibular variables measured on cephalograms. 
Table 4: displays the treatment changes and their significance in the maxillary/mandibular relationships as measured from cephalograms. The mean and standard deviation were calculated as well as paired t-test. ANB angle decreased significantly by average 2 degrees. In addition, the overjet also decreased significantly.

Table 4: Means and standard deviations as well as the paired $t$ value of the maxillary / mandibular relationship measured on cephalograms.

\begin{tabular}{|c|c|c|c|c|c|c|}
\hline \multirow{2}{*}{ Variables } & \multicolumn{2}{|c|}{ Pre-treatment } & \multicolumn{2}{c|}{ Post-treatment } & \multicolumn{2}{c|}{ Significance } \\
\cline { 2 - 7 } & Mean & S.D. & Mean & S.D. & T - Value & P $\leq$ \\
\hline ANB & 4.5 & \pm 0.43 & 2.5 & \pm 0.17 & 2.3 & $0.001^{* * *}$ \\
\hline Overjet & 5.86 & \pm 0.61 & 3.33 & \pm 0.25 & 9.2 & $0.001^{* * *}$ \\
\hline
\end{tabular}

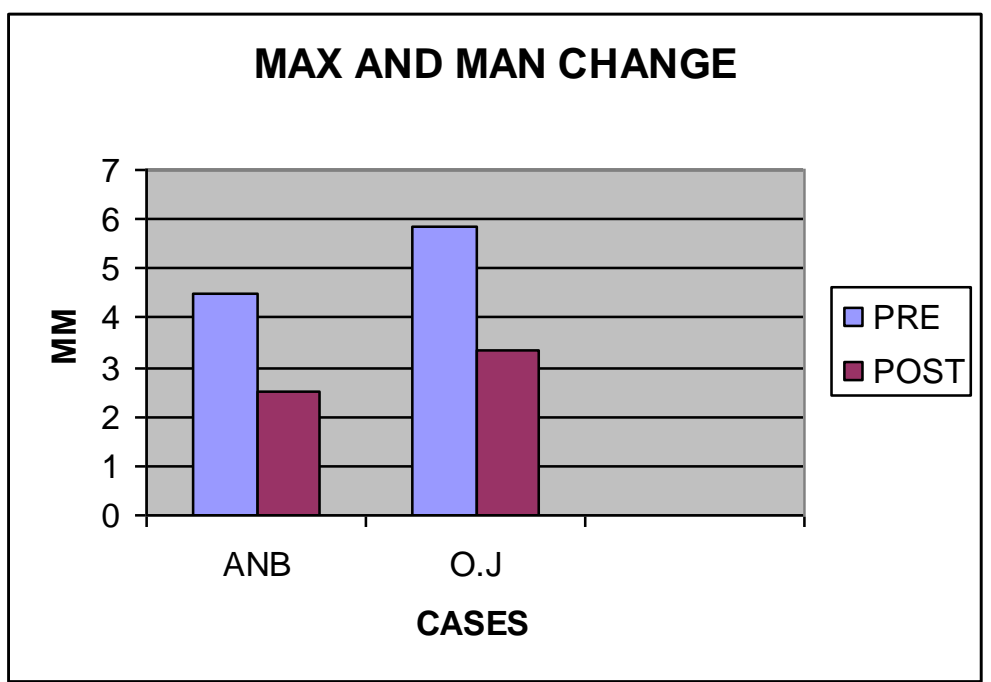

Fig. (5): Mean comparison of the maxillary / mandibular relationship measured on cephalograms.

Table 3 depicts the measurements to evaluate the maxillary/ mandibular relationships and treatment changes. Mean and standard deviation were calculated as well as paired t-test to display the treatment changes in the sample. In addition, the results were displayed graphically in figure 5 . 


\section{Egyptian}

Orthodontic Journal

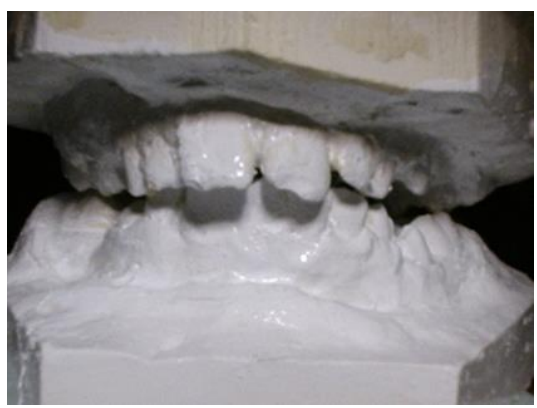

Fig. (6): Photograph of pre-treatment cast.

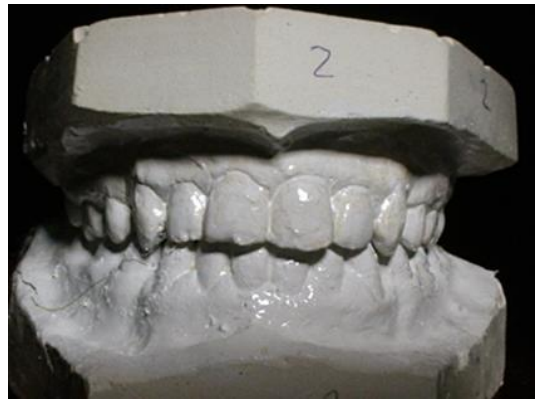

Fig. (7): Post-treatment cast.

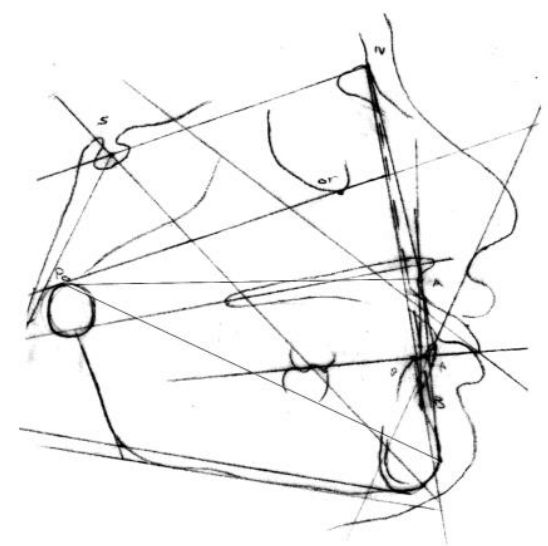

Fig. (9): Scanned tracing of the pretreatment cephalogram

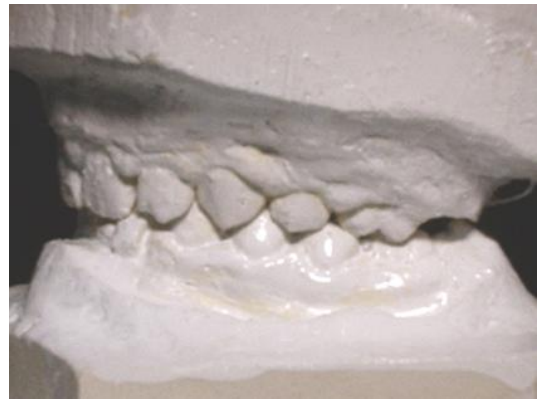

Fig. (8): Photograph of the posterior occlusion

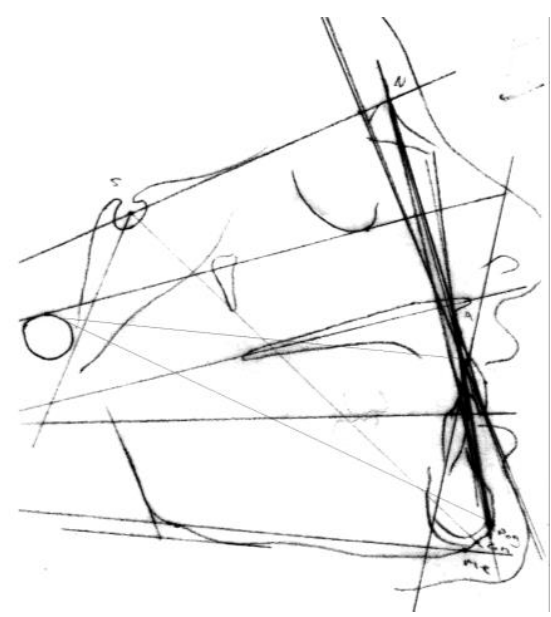

Fig. (10): Scanned tracing of the post treatment cephalogram. 


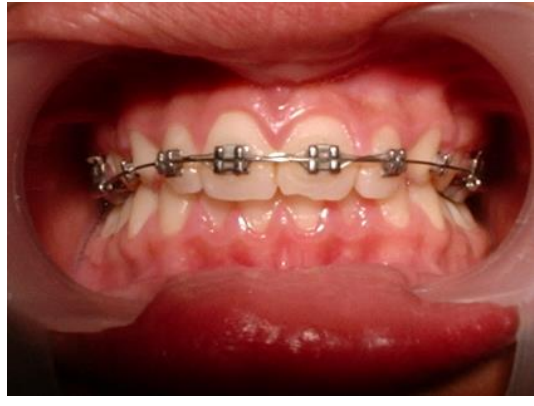

Fig. (11): Intra-oral photograph (during treatment).

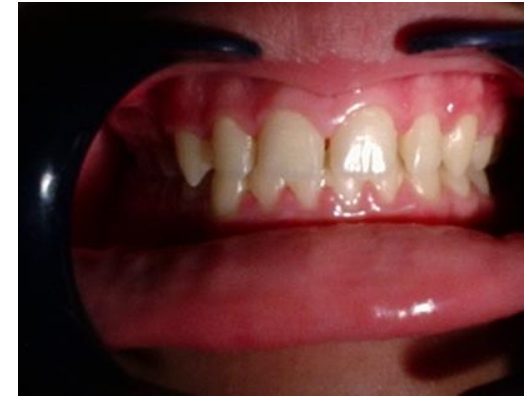

Fig. (12): Intra-oral post-treatment photograph.

\section{DISCUSSION}

The treatment of Class II malocclusion may be performed by extraction or non extraction. The extraction of four first premolars was more frequent than the extraction of maxillary first premolars and mandibular second premolars. Treatment by extraction of upper first premolars alone to correct canine relationship and camouflaging Class II malocclusion was rare, therefore direct comparison with other studies were not possible. The changes however, will be discussed descriptively and in relation with other ways of treatments.

In the present study a mean decrease in the maxillary arch perimeters was observed ( $\mathrm{t}$-value $=17.08$ ), and this change was expected due to extraction of upper first premolars and incisor retraction. This result was in agreement with the finding of Faten and Hala(14), Kaddah and Faten (15). On the other hand there was no significant change regarding the lower arch perimeter.

The increased value of the upper inter-canine width may be attributed to canines retractions to a wider widths to get class 1 canines relationship(14). This was true in the upper arch but was not observed in lower arch as the canines were not retracted. These results were in agreement with the previous finding of Thomas et al(10).

Other finding that should be evaluated and presented in this study was the decrease in the overjet after incisors retraction ( $t$-value $=12.7$ ).

One finding of this investigation was the decreased overbite and this can be explained by the intrusion of the upper anterior teeth during their retractions with the help of high pull headgears. In addition to the previous changes; the maxillary cephalometric variables were significantly decreased as a result of 
Egyptian

Orthodontic Journal

incisor retraction, changing the point $\mathrm{A}$, decreasing the effective maxillary length and significant change with retroclination of upper incisors relative to F.H plane. These changes were not significant for the lower arch except the relation of lower incisor to mandibular plane as there was some increase in this angle and this could be attributed to the nature of non extraction treatment. These findings were in agreement with the study of Germane et al(19), Bowman(20).

The maxillary/ mandibular relationships were also affected as the mean change was significantly decreased regarding ANB angle and overjet. These results could achieve the camouflaging of class II malocclusion and in agreement with the result of previous investigators $(9,21)$. Finally, we can conclude that the treatment of Class II Division 1 with uniarch extraction option could achieve compromise treatment with satisfactory results, as overjet and overbite were reduced. The canine relationship was corrected and Class II molar relationship was kept. The other aforementioned dental and skeletal changes were significant for the upper arch and not for the lower one. So, this treatment modality is indicated for patient with Class II division 1 with increased overjet and with aligned or minimal crowded lower arch to allow uniarch extraction treatment. Finally, the long-term studies of the obtained occlusion and its ,stability is recommended in future.

\section{REFERENCES}

1- Fouda M.A.: Disturbance of accepted occlusion by extraction. Al-Azhar Dental J, 1987; 5: 453-67.

2- El-Sayed H.S.: A clinical evaluation of different intraoral maxillary molar distalizing techniques. M.S. Thesis, Orthodontic Department, Cairo University, 2003.

3- Runge M.E., Martin J.T. and Bukai F.: Analysis of rapid maxillary molar distal movement without patient co-operation. Am J Orthod Dentofacial Orthop, 1999; 115: 153-57.

4- Marwa A.T: Dentoskeletal effect of the pendulum appliance. M.S Thesis, M.S Thesis, Orthodontic Department, Mansoura Faculty of Dentistry, 2006.

5- Fortini A, Lupoli M, Giuntoli F and Franchi L.: Dentoskeletal effects induced by rapid molar distalization with the first class appliance. Am J Orthod Dentofacial Orthop, 2004; 125:6697-704. 
6- Mesherry P. F. and Bradley H.: Class II Correction-Reducing patient Compliance: a Review of Available Techniques. J Clin Orthod. 2000; 27: 219-225 .

7- Proffit W.R.: Forty-year review of extraction frequencies at a university orthodontic clinic. Angle Orthod, 1994; 6: 407-414.

8- Conley S. R. and Jernigan C: Soft tissue changes after upper premolar extraction in Class II camouflage therapy. Angle Orthod, 2006; 76: 59-65.

9- Mihalik C. A., Proffit W. R. and Philips C.: long-term follow-up of Class II adult treated with orthodontic camouflage: a comparison with orthognathic surgery outcomes. Am J Orthod Dentofacial Orthop, 2003; 123: 3266-278.

10- Thomas D. Creekmore, DDS, Moderator; Norman M. Cetlin, DMD and Robert M. Rick: Roundtable: Diagnosis and Treatment Planning. J Clin Orthod, 1997; 992: $585-606$.

11- Bishara A. S., Bayati B., Zaher A. R. and Jakobsen J. R: Comparison of the dental arch changes in patients with Class II div. 1. extraction versus non extraction treatments. Angle Orthod., 1994; 5: 351-358.

12- Baum A. T.: Age and sex differences in the dentofacial changes following orthodontic treatment and their significance in treatment planning. Am J Orthod. 1961; 47: 355-362.

13- Dahan J, Serhal J.B. and Englebert A.: Cephalometric changes in Class II Division 1 cases after orthodontic treatment with bioactivator. Am J Orthod Dentofacial Orthop, 1989; 95: 127-137.

14- Faten H. E. and Hala M. S.: A clinical evaluation of dental arch changes and anchorage loss following mandibular canine retraction. Egypt Dental J, 2000; 46: 2327-2333.

15- Kaddah M. A. F. and Eid H. F.: Dentoskeletal and soft tissue changes associated with maxillary canine retraction. Egypt Dental J, 1999; 45: 4015-4022.

16- Mclaughlin R.P. and Bennett J. C.: The transition from standard edgewise to preadjusted appliance system. J Clin Orthod, 1989; 23: 142-53.

17- El-Mangoury H. Nagwa: Skeletal components of Angle Class II malocclusion. Egypt Orthod J, 1990; 4: 57-67. 
18- Maher A. Fouda, Ibrahim H. El-Kalla and Ahmed A. El-Bialy: Dentofacial growth changes of untreated Class II malocclusion; 3- Years study. Egypt Orthod J, 2001; 28:1-13.

19- Germane N, Líndauer S J, Rubenstein L K, Revere J H, and Isaacson R J: Increase in arch perimeter due to orthodontic expansion. Am J Orthod Dentofacial Orthop, 1991; 100, 421-427.

20- Bowman S. J.: Class II combination therapy. J Clin Orthod, 1998; 32:611-620.

21- Cetlin N. M. and Ten-Hoeve A: Nonextraction treatment. J Clin Orthod, 1983; 17:396 - 413 . 